\title{
Atom Counting in Atom Probe Tomography Specimens Using Quantitative HAADF- STEM
}

\author{
Williams Lefebvre
}

Université de Rouen, GPM, UMR CNRS 6634 BP 12, Avenue de l’Université 76801 Saint Etienne de Rouvray, France

Being able to provide the accurate position of atoms together with their chemical nature is a goal that is partially achieved by the Atom Probe Tomography (APT) technique. The reasons for this partial achievement are numerous and originate either from practical limitations related to the instrument (e.g. detection efficiency) or from the physics of field evaporation (e.g. trajectory aberrations). As a result, lattice resolution is rarely achieved by APT and a part of the original information contained in the specimen is reduced by the loss of ions during the detection. The specimen geometry and the evolution of this geometry during field evaporation also play a major role insofar as they impose reconstruction parameters which might evolve during the APT acquisition. Providing additional information about the evolution of specimen geometry would definitely help improving APT reconstructions and therefore lead to more accurate description of materials investigated. A correlative approach, which consists in combining APT with another characterization technique is hence suitable.

The choice of a relevant technique to be combined to APT is limited by several requirements. First, the technique must provide a three dimensional information about the specimen geometry. A simple projection or shadow image of the tip would hide the global disposition of emission sites along the surface, which knowledge is of relevance for the APT reconstruction. Second, the spatial resolution of the technique must be (ideally) close to $0.1 \mathrm{~nm}$ in order to reveal the details at the specimen surface that control the trajectories of ions emitted. Such a resolution would allow to observe local irregularities along the specimen surface at the origin of the local magnification artefact. Combined used of Scanning Transmission Electron Microscopy (STEM) or TEM is sometimes helpful for the improvement of reconstructions [1-2]. However, much more information can be obtained using Cs-probe corrected STEM since this technique already demonstrated the possibility of single atom detection and of 3D atomic imaging [3]. Indeed, this later technique routinely achieves spatial resolution below $0.1 \mathrm{~nm}$ and, following well defined protocols [4], enables atom counting from collected projections of crystals.

In the present work, we propose a method to reveal the three dimensional geometry of an APT specimen with an atomic resolution in STEM. For this purpose, we prepare sharp needle shape specimen of crystalline materials using focussed ion beam and we submit them to field evaporation in APT. Specimens are later introduced in the Cs-probe corrected STEM (JEOL AMR 200F) and observed in HAADF-STEM mode.

Atom counting from HAADF-STEM images is a non trivial issue. The reliability of such an approach strongly depends on the specimen thickness. Ideally, for a perfect zone axis orientation perpendicular to a low Miller index lattice plane, the electron probe is expected to travel along atomic columns parallel to the zone axis. This phenomenon is commonly referred to as the "channelling" of electrons. With increasing the specimen thickness, the probability that channelling electrons get trapped by neighbouring atomic columns increases, which gives rise to a "cross-talk" between columns. This artefact systematically needs to be considered as soon as a quantitative analysis of the HAADF signal is 
performed. In the present work, several sets of HAADF-STEM image simulations have been run in order to investigate the level of cross-talk as a function of specimen depth, for several sets of parameters defining the probe geometry. In addition, simulations were conducted on volumes corresponding to the particular geometry of APT specimens. All these simulations were conducted using the code developed by EJ Kirkland [5] in a version which includes the frozen phonon approximation. Based on HAADF image simulations, the variation of HAADF signal with the number of atoms is investigated (Figure 1). Atom counts in column are derived from experimental observations in tip shape specimens based on statistical treatment applied to recorded intensities, similar to those observed in Figure 2.
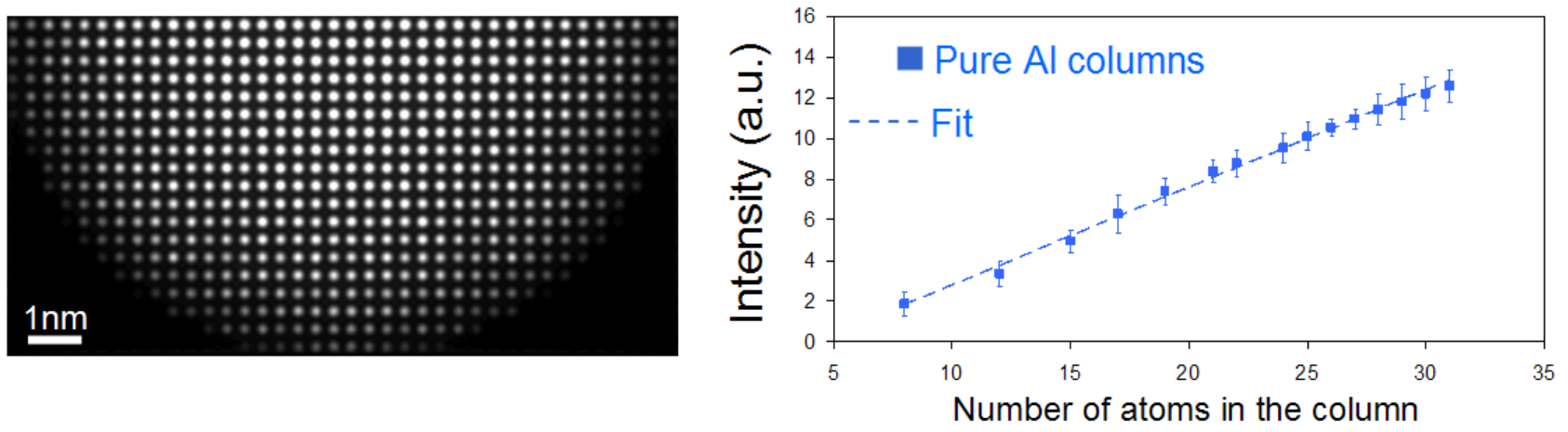

Figure 1. (Left) Simulated HAADF-STEM image of a tip shape Al crystal along [001] orientation. (Right) Relation between integrated atomic column intensity and number of atoms per column in the simulation.
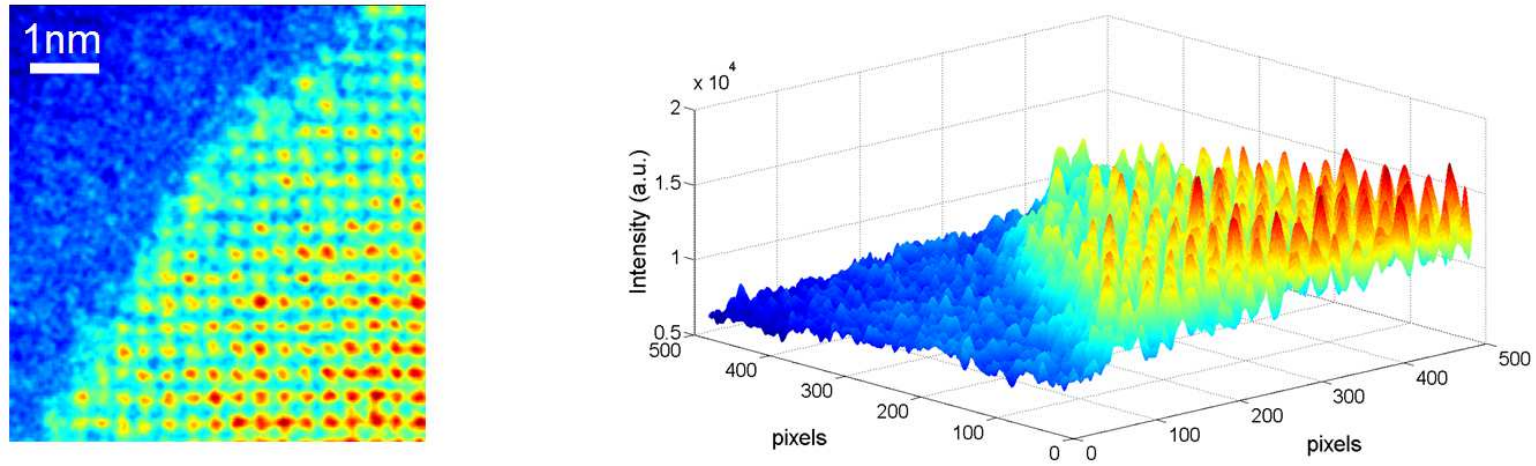

Figure 2. (Left) Selected region of an experimental HAADF-STEM image of an Al tip. (Right) Intensity distribution in the image.

[1] D Haley et al., J. of Mic. 244 (2011) 170.

[2] I Arslan et al., Ultramic. 108 (2008) 1579.

[3] S Van Aert, K.J. Batenburg, M.D. Rossell, R. Erni, G. Van Tendeloo, Nature 470, 374 (2011).

[4] S Van Aert, et al. Ultramicroscopy 109, 1236 (2009).

[5] J. Kirkland, Advanced Computing in Electron Microscopy, Plenum Press, New York (1998).

[6] The author gratefully acknowledge Pr. M. Robertson (Canadian Centre for Electron Microscopy) for providing an improved version of the code described in ref [5]. The ANR (Agence Nationale pour la Recherche) is thanked for the financial support through the Programme Jeune Chercheur - Jeune Chercheuse TIPSTEM. 